\title{
Ações virtuais de educação em saúde para população idosa: uma intervenção na comunidade
}

\author{
Virtual actions of health education for the elderly population: an intervention in the \\ community
}
Acciones virtuales de educación sanitaria para la población mayor: una intervención en la comunidad

Taiane Rocha da Silva Santos ${ }^{1 *}$, Cinthia Ravena Figueiredo Lira ${ }^{2}$, Luna Vitória Cajé Moura ${ }^{1}$, Luzia Lima dos Santos ${ }^{2}$, Kayck Mateus de Assis Santos ${ }^{1}$, Rafael Ribeiro Ramos ${ }^{1}$, Karinne da Silva Santos da Silva ${ }^{1}$, Neila Nayara Moreira de Oliveira ${ }^{1}$.

\section{RESUMO}

Objetivo: A presente pesquisa teve como intuito conhecer a percepção dos idosos de uma comunidade acerca da qualidade de vida, bem-estar e hábitos saudáveis. Métodos: Tratou-se de uma pesquisa, com abordagem qualitativa, do tipo exploratória e descritiva. Fizeram parte da pesquisa os idosos que são cadastrados em uma Unidade Básica de Saúde (UBS) do Recôncavo da Bahia. O primeiro momento do estudo aconteceu presencialmente no início de 2020, enquanto os demais momentos aconteceram por meio de intervenções virtuais. A análise ocorreu inicialmente com base na observação diagnóstica, pontuando através do discurso destas pessoas idosas. Posteriormente foram produzidos materiais em formato de podcasts, abordando as demandas apresentadas. Resultados: Os idosos consideravam qualidade de vida como sinônimo de vida e saúde em todos os aspectos, especificamente, saúde física, emocional e espiritual; quanto às limitações e manutenção dos hábitos, foi identificado dores na realização de atividades, comprometimento cognitivo e dificuldade em desapegar de hábitos adquiridos desde a infância. Conclusão: Notou-se que os idosos desta pesquisa possuíam um vasto conhecimento acerca do assunto e empenhavamse em adotar hábitos de vida que fossem saudáveis. Por outro lado, ficaram evidentes algumas dificuldades existentes na adoção destes hábitos, deixando claro o valor da educação em saúde.

Palavras-chave: Idoso, Educação em saúde, Qualidade de vida, Atenção primária à saúde.

\begin{abstract}
Objective: The present research aimed to know the perception of the elderly in a community about quality of life, well-being and healthy habits. Methods: This was a research, with a qualitative approach, exploratory and descriptive. The elderly who are registered in a Basic Health Unit (UBS) of Recôncavo da Bahia were part of the research. The first moment of the study took place in person at the beginning of 2020, while the other moments took place through virtual interventions. The analysis initially took place based on the diagnostic observation, punctuating through the speech of these elderly people. Subsequently, materials were produced in the form of podcasts, addressing the demands presented. Results: The elderly considered quality of life as synonymous with life and health in all aspects, specifically, physical, emotional and spiritual health; as for the limitations and maintenance of habits, pain was identified in the performance of activities, cognitive impairment and difficulty in letting go of habits acquired since childhood. Conclusion: It was noted that the elderly in this research had extensive knowledge about the subject and were committed to adopting healthy lifestyle habits. On the other hand, some difficulties in the adoption of these habits were evident, making clear the value of health education.
\end{abstract}

Keywords: Elderly, Health education, Quality of life, Primary health care.

\footnotetext{
${ }^{1}$ Faculdade Adventista da Bahia (FADBA), Cachoeira - BA. *E-mail: tayrocha.1717@gmail.com

2 Hospital Adventista Silvestre, Rio de Janeiro - RJ.
} 


\section{RESUMEN}

Objetivo: La presente investigación tuvo como objetivo conocer la percepción de las personas mayores en una comunidad sobre la calidad de vida, el bienestar y los hábitos saludables. Métodos: Esta fue una investigación, con un enfoque cualitativo, exploratorio y descriptivo. Los ancianos que se encuentran registrados en una Unidad Básica de Salud (UBS) de Recôncavo da Bahia fueron parte de la investigación. El primer momento del estudio tuvo lugar presencialmente a principios de 2020, mientras que los demás momentos se desarrollaron a través de intervenciones virtuales. El análisis se realizó inicialmente a partir de la observación diagnóstica, puntuándose a través del habla de estas personas mayores. Posteriormente, se produjeron materiales en forma de podcasts, atendiendo las demandas presentadas. Resultados: Los adultos mayores consideraron la calidad de vida como sinónimo de vida y salud en todos los aspectos, específicamente, salud física, emocional y espiritual; En cuanto a las limitaciones y mantenimiento de hábitos, se identificó dolor en la realización de actividades, deterioro cognitivo y dificultad para dejar de lado los hábitos adquiridos desde la infancia. Conclusión: Se observó que los adultos mayores de esta investigación tenían un amplio conocimiento sobre el tema y estaban comprometidos con la adopción de hábitos de vida saludables. Por otro lado, se evidenciaron algunas dificultades en la adopción de estos hábitos, dejando claro el valor de la educación para la salud.

Palabras clave: Ancianos, Educación sanitaria, Calidad de vida, Atención primaria de salud.

\section{INTRODUÇÃO}

A população mundial está envelhecendo, este é um fato incontestável. O envelhecimento populacional é considerado um triunfo da humanidade e também um dos maiores desafios da saúde pública contemporânea. Considera-se que a velhice tem início aos 65 anos nos países desenvolvidos e aos 60 anos nos países em desenvolvimento (SERBIM AK, et al., 2013).

Pesquisas apontam, que é nos países em desenvolvimento que o envelhecimento da população tem ocorrido mais intensamente. No Brasil, o número de idosos ( $\geq 60$ anos de idade) passou de 3 milhões em 1960, para 7 milhões em 1975, e 14 milhões em 2002 (um aumento de 500\% em quarenta anos) e deverá alcançar 32 milhões em 2020 (VERAS RP e OLIVEIRA M, 2018).

A população idosa apresenta um alto grau de incidência de morbidades crônicas tais como diabetes, doenças neurodegenerativas, hipertensão arterial, entre outras, que comprometem de forma recorrente a realização de atividades comuns da vida diária (AVD) (POLTRONIERI BC, et al., 2019). Porém Gautério DP, et al., (2014), salientam que é possível afirmar que alguns fatores determinam a forma como os indivíduos irão envelhecer, tais como estilo de vida, sedentarismo, estresse, acidentes, comorbidades, dentre outros.

A fim de obter melhores condições de vida, com consequente redução do número de agravos que poderiam requerer tratamento curativo, a proposta de promoção à saúde, voltada para educação em saúde, configura-se como ferramenta de intervenção pedagógica bem-sucedida (CABRAL JR, 2015). Os achados dos autores Seabra CA, et al. (2019), colocam em evidência o valor da educação em saúde para essa população específica, principalmente quando ocorre a troca de saberes científico e popular.

Ademais, acredita-se que a realização de intervenções virtuais de Educação em Saúde com a população idosa na atenção primária pode contribuir para uma melhor qualidade de vida, bem-estar e hábitos saudáveis. Para o manejo efetivo dessa população, o autor Mendonça FT, et al. (2017), discorre que a educação em saúde é parte destacada das atribuições dos profissionais da atenção primária à saúde.

Evidencia-se, portanto, relevância de estudos que abordem esta temática de educação em saúde para a população idosa. Visto que, segundo Souza EM, et al. (2019), estudar sobre a temática pode ajudar na descoberta das principais dificuldades e dúvidas apresentadas pela população idosa para a adção e manutenção de hábitos saudáveis, bem como conhecer os fatores que interferem para a cessação de hábitos maléficos. Todos estes saberes devem servir de subsídio para que a equipe multiprofissional em saúde esteja munida em prol do bem estar das pessoas idosas.

Assim sendo, a presente pesquisa teve como intuito conhecer a percepção dos idosos de uma comunidade acerca da qualidade de vida, bem-estar e hábitos saudáveis, para que a partir disso, fossem desenvolvidas intervenções virtuais, conforme as demandas relatadas por eles. 


\section{MÉTODOS}

Trata-se de uma pesquisa de abordagem qualitativa, do tipo exploratória e descritiva. Participaram da pesquisa os idosos que são cadastrados em uma Unidade Básica de Saúde (UBS) do Recôncavo da Bahia. O primeiro encontro aconteceu de forma presencial na casa de cada um dos participantes, para que fosse feito o diagnóstico situacional, enquanto que os demais momentos ocorreram de forma online.

Sendo respeitados os aspectos éticos e legais, consoante às orientações contidas na Resolução de no 510/2016- Comissão Nacional de Ética em Pesquisa (CONEP/MS), conforme parágrafo III que orienta sobre: "respeito aos valores culturais, sociais, morais e religiosos, bem como aos hábitos e costumes, dos participantes das pesquisas" (BRASIL, 2016). Ao passo em que foi aprovado pelo Comitê de ética em pesquisa.

A coleta de dados contou com o auxílio de um roteiro, elaborado especificamente para este fim, contendo perguntas norteadoras e pertinentes, que dizem respeito ao estilo de vida do idoso, seus hábitos de cuidado em saúde no cotidiano e suas percepções sobre as atividades de educação em saúde de modo geral.

Aos idosos participantes foram assegurados da confiabilidade, o anonimato e a confidencialidade das informações coletadas, de forma que os dados obtidos não foram associados à sua pessoa no momento da leitura do trabalho a partir da atribuição de pseudônimos aos envolvidos. Sendo gravado em um aparelho celular todas as falas dos participantes, e os dados coletados foram analisados e registrados, levando em consideração o sigilo total da identificação dos participantes da pesquisa.

Após o diagnóstico situacional, foi feita uma avaliação de resultados para identificar possíveis problemas e então realizar as intervenções virtuais com base no que fora encontrado. Nestas intervenções virtuais foram enviados via WhatsApp vários podcasts e vídeos interativos, abordando as principais demandas por eles apresentadas.

A análise ocorreu inicialmente com base na observação diagnóstica, pontuando através do discurso destas pessoas idosas, o grau de conhecimento que possuem sobre as práticas favoráveis à saúde, bem-estar e hábitos saudáveis. Posteriormente, ao término das intervenções virtuais, foi produzido um relatório a partir das questões levantadas e dos discursos apreendidos pelas falas dos idosos participantes, após sua transcrição.

Através dos núcleos de sentido emergidos a partir da fala dos participantes, tendo como base a análise de conteúdo temática proposta por Bardin L (2011), as pesquisadoras separaram em categorias e subcategorias os resultados obtidos nestes discursos.

Deste modo, descobriu-se qual a percepção e o grau de conhecimento que possuíam sobre as práticas favoráveis à saúde, bem-estar e hábitos saudáveis, para que fossem então, desenvolvidas as intervenções virtuais, conforme as demandas relatadas por eles, tendo em foco uma modificação desta realidade.

\section{RESULTADOS E DISCUSSÃO}

Mediante a coleta de dados foi possível obter um perfil dos participantes, os mesmos apresentavam faixa etária entre 60 a 83 anos de idade, participaram da pesquisa um total de 12 idosos, no qual em sua maioria está representado por um número superior de mulheres, sendo onze mulheres e um homem. É importante destacar que todos estes idosos, eram profundamente religiosos e viviam em uma comunidade circunvizinha a uma Instituição de ensino superior religiosa que desempenha atividades educativas e sociais frequentes na comunidade.

As principais questões norteadoras que foram utilizadas no roteiro usado na coleta de dados, estão relacionadas à qualidade de vida, prática de hábitos saudáveis, dificuldades em adotar hábitos saudáveis e principais dúvidas relacionadas à saúde do idoso. Salienta-se que este questionário foi respondido exclusivamente pelos próprios idosos, ao passo em que tiveram disponíveis a todo instante o suporte das pesquisadoras para auxiliar naquilo que necessário fosse. 
Sobre as comorbidades, foi possível identificar quais estiveram mais prevalentes, dentre elas estão: Hipertensão Arterial (HAS), diabetes, colesterol e aspectos relacionados à saúde mental. Que corrobora com uma pesquisa encontrada, onde diz que: "A população idosa apresenta um alto grau de incidência de morbidades crônicas tais como diabetes, doenças neurodegenerativas, hipertensão arterial, entre outras, que comprometem de forma recorrente a realização de atividades comuns da vida diária (AVD) " (POLTRONIERI BC, et al., 2019).

Com base no discurso dos idosos emergiu uma categoria para posterior análise, sendo ela: Categoria qualidade de vida, saúde e hábitos saudáveis na terceira idade: o que conhecem e o que praticam. Contendo nesta, três subcategorias: Subcategoria 1- percepções acerca da qualidade de vida, saúde e hábitos saudáveis. Subcategoria 2- fatores intervenientes para a adoção ou manutenção de hábitos saudáveis. Subcategoria 3- dificuldades e dúvidas acerca da adoção ou manutenção de hábitos saudáveis.

\section{Qualidade de vida, saúde e hábitos saudáveis na terceira idade: o que conhecem e o que praticam: \\ Percepções acerca da qualidade de vida, saúde e hábitos saudáveis}

Foi possível observar que os idosos da presente pesquisa, consideram qualidade de vida como sinônimo de vida, de querer viver e ter saúde em todos os aspectos, especificamente, saúde física, emocional e espiritual, buscando adotar a prática de hábitos saudáveis, baseando-se nos "oito remédios da natureza".

Segundo White EG (2013), uma escritora cristã norte-americana e uma das fundadoras da Igreja Adventista do Sétimo Dia, considerada uma das escritoras mais significantes da literatura americana,um conjunto de práticas que tem como objetivo final ensejar uma maior qualidade de vida, denominado em 1863 como os oito remédios naturais (ORN), é formado por água, ar puro, confiança em Deus, descanço, exercício físico, luz solar, nutrição e temperança. As intervenções em saúde que implementam os ORN são vistas como imprecindíveis no que se refere a promoção de saúde e qualidade de vida.

Justifica-se então, pelos idosos participantes da pesquisa serem representados em sua maior parte por idosos religiosos que acreditavam veementemente na eficácia destes hábitos para se alcançar uma melhor qualidade de vida.

Ao se questionar sobre o que entendiam por qualidade de vida e hábitos saudáveis no envelhecimento, apontaram que:

"Os hábitos saudáveis "é" dormir cedo, ter horário certo para alimentação, não comer muito, comunhão e ter o hábito de orar [...]” (Idoso 3).

"É querer viver, tendo uma saúde completa, uma saúde mental, emocional e espiritual" (Idoso 5).

"Qualidade de vida na terceira idade é buscar os remédios naturais, duração do sono, tomar água [...]" (Idoso 7).

"[...] qualidade de vida é ter saúde em todos os pontos [...]" (Idoso 8).

"É ter uma alimentação equilibrada, tomar muita água, fazer caminhada, exercício físico né e seguir os oito remédios naturais [...]" (Idoso 9).

É possível observar que para os idosos entrevistados a uma estreita relação entre ter qualidade de vida e seguir as orientações relativas aos "oito remédios naturais". É importante destacar que o tema dos "oito remédios naturais" é oriundo de literaturas essencialmente religiosas, logo nota-se que para tais idosos os ensinamentos religiosos são a base do que se compreende por qualidade de vida. White EG (2013), comenta sobre os oito remédios naturais, onde fala que muitas enfermidades poderiam ser evitadas ou curadas se os homens praticassem tão simples orientações.

Corroborando com uma pesquisa realizada, onde os autores descrevem a importância de priorizar o cuidado do ser humano, do ponto de vista integral, resgatando os valores subjetivos e respeitando os aspectos espirituais e religiosos que cada um carrega consigo, isto servindo de alicerce para a tomada de decisões (NUNES MG, et al., 2017). 
Nota-se também, que ao tratar dos meios pelos quais é possível obter qualidade de vida, os idosos se referiram a práticas objetivas e perfeitamente aplicáveis, o que demonstra que para eles ter qualidade de vida é mais que uma questão subjetiva ou uma realidade inacessível, pelo contrário, todos os meios apresentados apontam para uma qualidade de vida que se pode obter por recursos naturais, e em sua maioria, gratuitos.

\section{Fatores intervenientes para a adoção ou manutenção de hábitos saudáveis}

Dentro desse perfil, evidenciaram-se as principais dificuldades que os idosos possuíam em relação à pratica constante de hábitos saudáveis, dentre elas destacaram- se as limitações consequentes da idade e agravos de comorbidades. As dores que sentem durante a realização de exercícios físicos, até mesmo, ao realizarem atividades consideradas simples do cotidiano, comprometimento cognitivo afetado, autocontrole na alimentação e o desapego de maus hábitos adquiridos desde a infância.

Sobre as dores durante atividades, pontuaram que buscam se esforçar para não terem um estilo de vida sedentário, porém sentem muitas dificuldades ao praticarem exercícios físicos, até mesmo uma simples caminhada, por não aguentarem ir muito longe, assim como, apresentam também limitações no desempenho das suas atividades diárias.

Estudos mostram que há inúmeros fatores que podem contribuir para as limitações presentes na vida do idoso. A maior prevalência de inatividade física entre idosos longevos pode ser explicada pela presença de barreiras como limitação física, medo de quedas, presença de dores, falta de disposição, falta de segurança (RIBEIRO AQ, et al., 2016).

"Eu não aguento ficar dez minutos em pé, não acompanhava os exercícios eu não aguentei, é muita dor mesmo, Além do joelho, eu pesada desse jeito [...]" (Idoso 2).

"[...] quebrei o fêmur, aí me limita em alguma coisa, porque por exemplo, agora eu não sento em lugares baixo, por causa da prótese, aí existe uma certa limitação, mas antes eu fazia tudo [...]" (Idoso 8).

Acerca do comprometimento cognitivo apontaram que nesta fase da terceira idade, o raciocínio encontrase consideravelmente mais lento, interferindo assim na memorização de informações necessárias para o bom convívio familiar e na sociedade onde vivem.

Em um determinado estudo identificou-se que a memória, por ter um papel fundamental no processo de ensino-aprendizagem e na definição do indivíduo sobre sua identidade, constitui uma das funções cognitivas essenciais do ser humano. Sem ela, não seria possível, por exemplo, acumular e transmitir experiências a outras pessoas, o que promove a socialização da espécie humana (CASEMIRO FG, et al., 2017).

Os achados dos autores Casemiro FG, et al. (2017), descrevem que a manutenção da cognição é relevante para a promoção da independência e autonomia do idoso e, portanto, estimulá-la pode prevenir ou mesmo retardar o processo de degeneração do cérebro.

Deste modo, considera-se que o bom funcionamento cognitivo seja imprescindível para que o idoso continue vivendo de maneira independente e consequentemente tendo uma maior qualidade de vida. Portanto, existe a necessidade de trabalhá-la e estimulá-la constantemente para uma melhoria neste processo.

\section{"[...] o raciocínio é muito mais lento [...]" (Idoso 4). \\ "[...] também a questão da mente, que precisa exercitar a mente, tem que exercitar os neurônios [...]" (Idoso 7).}

Por fim, outro fator que referiram interferir na adoção ou manutenção de hábitos saudáveis foi a dificuldade em desapegar de hábitos adquiridos desde a infância, pois os mesmos refletem significativamente no estilo de vida que possuem atualmente, ousa-se dizer que em todos os aspectos da vida, todavia especialmente nos hábitos alimentares.

As narrativas desvelam as particularidades que permeiam a manutenção de hábitos saudáveis: 
"Principalmente quando se tem por exemplo, pressão alta aí tem que controlar o sal o açúcar e isso aí é difícil, comer ainda mais sem sal não dá, é muito difícil [...]. Tem o problema da mente que precisa exercitar a mente, tem que exercitar os neurônios" (Idoso 7).

"Não consegui vencer ainda esse habito de tomar café, minha vó era viciada em café estou correndo a todo tempo para eu me lagar [...]" (Idoso 9).

"O que dificulta é que desde lá da minha infância que eu já fui criada assim nesse ritmo [...]" (Idoso 12).

\section{Dificuldades e dúvidas acerca da adoção ou manutenção de hábitos saudáveis}

Parte dos participantes relatou a existência de conflitos de informações recebidas acerca da saúde.

"Realmente eu tenho muita dúvida, por que os próprios médicos, um diz uma coisa, outro diz outra [...]" (Idoso 1).

Por outro lado, grande parte dos participantes idosos, disseram não haver dúvidas porque leem muito e buscam informações na internet quando apresentam dúvidas em relação algum assunto que diz respeito a saúde.

Este contexto se mostra então como um ponto positivo, pois esse público acaba tendo mais acesso a informações. Coutinho C e Lisboa $\mathrm{E}$ (2011), comentam que a sociedade atual vem se caracterizando como a sociedade da informação, dominada por tecnologias digitais como a internet. É possível dizer que a velhice pode ser vista como uma fase de desenvolvimento, sendo descrita por vários estudos como uma fase repleta de possibilidades, como qualquer outra, no qual o idoso pode desfrutar de uma velhice bem-sucedida e com qualidade de vida.

Além disso, alegam que participam de atividades e ouvem diversas palestras ofertadas pelos acadêmicos da faculdade próxima à comunidade em que estão inseridos, sentem-se privilegiados pelos os estudantes sempre promoverem eventos que os incluam. Eles fizeram questão de deixar claro que estão abertos e que gostam de aprender sempre mais, pois conforme os mesmos, o conhecimento nunca é demais.

"Olha, eu estou sempre orando a Deus, ouvindo-a novo tempo (uma emissora evangélica de rádio e televisão) ensinamentos, as palestras que tem na faculdade, eu sempre quero aprender mais [...]" (Idoso 6).

"Não, porque é o seguinte, eu leio muito, sei tudo quanto é remédio [...]" (Idoso 3).

"[...] a gente lê muito né, é aquela coisa, ainda mais aqui que a gente participa de muitas coisas aqui na faculdade" (Idoso 7 ).

Mesmo conhecendo todos os benefícios que uma alimentação saudável, bem como a prática regular de atividade física para a promoção da saúde, muitos brasileiros apresentam dificuldades em aderir a estilos de vida mais saudáveis (TOLEDO MT, et al., 2013).

A partir dos discursos dos participantes da pesquisa foram implementadas algumas intervenções virtuais e enviadas via WhatsApp, sendo elas: 12 podcasts, elaborados por graduandos de enfermagem, com temas relacionados à saúde do idoso e um vídeo interativo, falando sobre a importância da alimentação saudável, apresentando uma receita bem prática de um suco natural, o vídeo teve duração de 20 minutos e todos os conteúdos foram abordando as principais demandas por eles apresentadas.

Existiram momentos de diálogo com os participantes via WhatsApp, foi feito o levantamento de problemas através destes diálogos e busca por soluções, ou atitudes que os amenizem, por acreditar que estes momentos contribuam substancialmente para a produção de conhecimento e modificação da realidade. Deixando claro o valor da educação em saúde, visto que é potencialmente importante para a transformação dos modos de vida dos indivíduos e da coletividade e, consequentemente, promover qualidade de vida e saúde. 
Corroborando com os achados dos autores Seabra CA, et al. (2019), que evidenciam o valor da educação em saúde para essa população específica, principalmente quando ocorre a troca de saberes científico e popular; valorizando o saber mútuo, dando importância ao diálogo e elevando o poder de compreensão do idoso de si, do outro e do mundo, ampliando a compreensão de realidades diversas. Deste modo, iniciativas como a em tela se mostram pertinentes, e seu impacto social de considerável relevância.

Após a realização das intervenções educativas online, os idosos participantes relataram contentamento com as informações trocadas, pontuaram descobrir sobre muitos assuntos que entendiam superficialmente. Também relatarm que fizeram o envio dos materiais recebidos para familiares e outras pessoas idosas.

\section{CONCLUSÃO}

O presente estudo permitiu a ampliação do entendimento sobre a percepção dos idosos de uma comunidade acerca da qualidade de vida, bem-estar e hábitos saudáveis, a partir disso, foi possível desenvolver as intervenções virtuais, mediante as demandas apresentadas. Algumas limitações deste estudo dizem respeito ao fato de não conseguir acompanhar a longo e médio prazo os impactos das intervenções virtuais na vida dos idosos, quais foram suas influências e contribuições, por não ter tido um tempo hábil para o acompanhamento e repercussão do estudo. Entretanto, tal limitação não se configurou como demérito ao estudo em tela, e se mostra como uma interessante lacuna a ser explorada em estudos futuros sobre a temática.

\section{REFERÊNCIAS}

1. BARDIN L. Análise de conteúdo. 4ª ed. revista e atualizada. Lisboa: Edições 70, 2011. 281 p.

2. BRASIL. Manual do Ministério da Saúde, 2016. Disponível em: < https://conselho.saude.gov.br/resolucoes/2016/Reso510.pdf >. Acesso em 27 de março de 2020.

3. CABRAL JR, et al. Oficinas de educação em saúde com idosos: uma estratégia de promoção da qualidade de vida. Revista Enfermagem Digital Cuidado e Promoção da Saúde, 2015; 1: 71-75.

4. CASEMIRO FG, et al. Efeitos da educação em saúde em idosos com comprometimento cognitivo leve. Revista Brasileira de Enfermagem REBEN, 2018; 71: 854-63.

5. CIOLA G, et al. Dor crônica em idosos e associações diretas e indiretas com variáveis sociodemográficas e de condições de saúde: uma análise de caminhos. Rev. Bras. Geriatr. Gerontol, 2020; 23(3): e200065.

6. COUTINHO C, LISBOA E, Sociedade da informação, do conhecimento e da aprendizagem: desafios para educação no século XXI. Revista de Educação, 2011; 18: 5-22.

7. CUNHA P, PINHEIRO LC, O papel do exercício físico na prenvenção das quedas nos idosos: Uma revisão baseada em evidência. Rev Port Med Geral Fam., 2016; 32: 96-100.

8. FLORES TR, et al. Hábitos saudáveis: que tipo de orientação a população idosa está recebendo dos profissionais de saúde?. REV BRAS EPIDEMIOL., 2016; 19(1): 167-180.

9. GAUTÉRIO DP, et al. Ações educativas do enfermeiro para a pessoa idosa: estratégia saúde da família. Revista Enfermagem UERJ, 2014; 21: 824-828.

10. GOMES AP, et al. Padrões alimentares de idosos e seus determinantes: estudo de base populacional no sul do Brasil. Ciência \& Saúde Coletiva, 2020; 25(6): 1999-2008, 202.

11. LEÃO OA, et al. Comportamento sedentário em idosos residentes de zona rural no extremo Sul do Brasil. REV BRAS EPIDEMIOL, 2020; 23: e200008.

12. MENDONÇA FT, et al. Educação em saúde com idosos: pesquisa-ação com profissionais da atenção primária. Rev. Bras. Enferm. Brasília, 2017; 70.

13. NUNES MG, et al. Idosos longevos: avaliação da qualidade de vida no domínio da espiritualidade, da religiosidade e de crenças pessoais. Saúde debate,2017; 41: 1102-1115.

14. POLTRONIERI BC, et al. Análise do tema violência nas políticas de cuidado de longa duração ao idoso. Ciência e Saúde coletiva, 2019; 24: 2859-2869.

15. RIBEIRO AQ, et al. Prevalência e fatores associados à inatividade física em idosos: um estudo de base populacional. Rev. Bras. Geriatr. Gerontol, 2016; 19: 483-493.

16. SEABRA CA, et al. Educação em saúde como estratégia para promoção da saúde dos idosos: Uma revisão integrativa. Rev. bras. geriatr. gerontol, 2019; 22(4).

17.SERBIM AK, et al. Oficinas Multiprofissionais: Educação em saúde para idosos de uma comunidade. Revista Eletrônica Gestão e Saúde, 2013; 4: 1500-1510.

18. SOUZA EM, et al. Educação popular, promoção da saúde e envelhecimento ativo: uma revisão bibliográfica integrativa. Revista ciêcia e saúde coletiva. 2019; 9(6).

19. TOLEDO MT, et al. Adesão a modos saudáveis de vida mediante aconselhamento por profissionais de saúde. Rev. Saúde Pública, 2013; 47: 540-548.

20. VERAS RP, OLIVEIRA M. Envelhecer no Brasil: a construção de um modelo de cuidado. Rev. Ciência e Saúde Coletiva, 2018; 23: 61929-1936.

21. WHITE EG, A ciência do bom viver. Estados Unidos: Copyright, 2013. 378 p. 